Article

\title{
Biofabrication of Cell-Derived Nanovesicles: A Potential Alternative to Extracellular Vesicles for Regenerative Medicine
}

\author{
Nazma F. Ilahibaks ${ }^{1, \dagger}$, Zhiyong Lei ${ }^{1,2,+}{ }^{+}$Emma A. Mol ${ }^{1}$, Anil K. Deshantri ${ }^{2} \mathbb{(}$, Linglei Jiang ${ }^{2}$, \\ Raymond M. Schiffelers ${ }^{2}$, Pieter Vader ${ }^{1,2}$ and Joost P.G. Sluijter ${ }^{1,3, *(\mathbb{D}}$ \\ 1 Laboratory of Experimental Cardiology, Department of Cardiology, University Medical Center Utrecht, \\ 3584 CX Utrecht, The Netherlands; n.f.ilahibaks-2@umcutrecht.nl (N.F.I.); z.lei@umcutrecht.nl (Z.L.); \\ E.A.Mol-5@umcutrecht.nl (E.A.M.); pvader@umcutrecht.nl (P.V.) \\ 2 Department of Clinical Chemistry and Hematology, University Medical Center Utrecht, 3584 CX Utrecht, \\ The Netherlands; anildeogarh@gmail.com (A.K.D.); L.Jiang-2@umcutrecht.nl (L.J.); \\ R.Schiffelers@umcutrecht.nl (R.M.S.) \\ 3 Circulatory Health Laboratory, Regenerative Medicine Center, University Medical Center Utrecht, \\ University Utrecht, 3584 CX Utrecht, The Netherlands \\ * Correspondence: J.Sluijter@umcutrecht.nl \\ + These authors contributed equally to this work.
}

Received: 1 November 2019; Accepted: 20 November 2019; Published: 25 November 2019

\begin{abstract}
Extracellular vesicles (EVs) are mediators of intercellular communication by transferring functional biomolecules from their originating cells to recipient cells. This intrinsic ability has gained EVs increased scientific interest in their use as a direct therapeutic in the field of regenerative medicine or as vehicles for drug delivery. EVs derived from stem cells or progenitor cells can act as paracrine mediators to promote repair and regeneration of damaged tissues. Despite substantial research efforts into EVs for various applications, their use remains limited by the lack of highly efficient and scalable production methods. Here, we present the biofabrication of cell-derived nanovesicles (NVs) as a scalable, efficient, and cost-effective production alternative to EVs. We demonstrate that NVs have a comparable size and morphology as EVs, but lack standard EV (surface) markers. Additionally, in vitro uptake experiments show that human fetal cardiac fibroblast, endothelial cells, and cardiomyocyte progenitor cells internalize NVs. We observed that cardiac progenitor cell-derived NVs and EVs are capable of activating mitogen-activated protein kinase 1/2 (MAPK1/2)-extracellular signal-regulated kinase, and that both NVs and EVs derived from A431 and HEK293 cells can functionally deliver Cre-recombinase mRNA or protein to other cells. These observations indicate that NVs may have similar functional properties as EVs. Therefore, NVs have the potential to be applied for therapeutic delivery and regenerative medicine purposes.
\end{abstract}

Keywords: extracellular vesicles; biofabrication; cell-derived nanovesicles; therapeutic delivery; regenerative medicine

\section{Introduction}

Extracellular vesicles (EVs) are cell-derived nanoparticles consisting of a lipid bilayer encapsulating cytosolic content from their cell of origin. EVs can exchange information between cells by delivering biomolecules, e.g., proteins, nucleic acids, or lipids, from their parent cells to target cells [1]. EVs' endogenous origin, low immunogenicity, intrinsic targeting abilities, and biocompatibility are potential advantages features compared to conventional drug delivery systems such as liposomes, polymers, and gold particles [2-4]. 
EVs are also considered as a cell-free therapy for regenerative medicine since various studies have shown that progenitor cells exert their therapeutic reparative benefit via a paracrine mechanism in which EVs play a fundamental role [2-11]. In cardiovascular disease, human fetal cardiac progenitor cell (CPC)-derived EVs stimulate pro-angiogenic effects and improve cardiac function upon injury [12,13]. Therefore, $\mathrm{CPC}-\mathrm{EV}$ s are an attractive therapeutic candidate to stimulate cardiac repair and regeneration, since the heart has a very poor endogenous regenerative capacity [14-18].

Despite EVs' potential, their application is restrained due to the lack of highly efficient, scalable, and cost-effective production and isolation methods [19]. Large amounts of cells and culture medium, as well as time-consuming and labor-intensive isolation and purification processes, are required to obtain substantial quantities of EVs $[20,21]$. Various approaches have been investigated to augment the production of EVs [21-25]. However, these studies either do not show data indicating whether their approach yields considerable amounts of EVs, save time in their production or isolation processes, or demonstrate functional delivery of EVs.

Hence, this study aimed to investigate the spontaneous self-assembly of cell-derived vesicles (NVs), so-called biofabrication of NVs, as an alternative production method to make EV mimetics. During NV biofabrication, cells are subjected to sonication followed by sequential extrusion through microfilters to yield nanosized vesicles (Figure 1a). We hypothesized that during the NV biofabrication process, the membrane compositions of parental cells would remain, while their cytosolic content would partially be encapsulated in the lumen of NVs.

We found that biofabrication of NVs is a scalable and efficient alternative of EV production to obtain a high yield of cell-derived nanosized vesicles. We also demonstrated that NVs are capable of transferring functional biological material to recipient cells and can activate intracellular pro-survival signaling pathways. To the best of our knowledge, this is the first report on biofabrication of CPC-NVs that demonstrates the induction of phosphorylation of mitogen-activated protein kinase 1/2 (MAPK1/2)-extracellular signal-regulated kinase (ERK1/2) and the active delivery of Cre-recombinase mRNA/protein into target cells. These observations indicate that NVs have the potential to be further developed as a potential alternative for CPC-EVs in regenerative medicine or be engineered for drug delivery purposes.

\section{Materials and Methods}

\subsection{Cell Culture}

Human embryonic kidney cells 293T (HEK293T), 293FT cells (HEK293FT), and human fetal cardiac fibroblasts (CF) were cultured in Dulbecco's Modified Eagle Medium (DMEM) (41965039, Gibco, Paisley, Scotland, UK) supplemented with 10\% (v/v) fetal bovine serum (FBS) and 1\% (v/v) penicillin-streptomycin $(\mathrm{P} / \mathrm{S})$. Human fetal heart tissue was obtained by individual permission via a standard written informed consent procedure following the Declaration of Helsinki and approval of the ethics committee of the Leiden University Medical Center (LUMC), The Netherlands. The subsequently obtained human cardiac-derived progenitor cells were provided anonymously via an MTA from LUMC, The Netherlands. CPC and human microvascular endothelial cells (HMEC-1) were obtained and cultured in appropriate cell culture media, as previously described $[13,26,27]$. Human umbilical vein endothelial cells with a cytosolic green fluorescent protein (HUVEC-GFP) were obtained and cultured, as described previously [13]. A431-Cre, HEK293FT-Cre donor cells, and T47D-stoplight reporter cells were cultured in DMEM (41965039, Gibco, Paisley, Scotland, UK) supplemented with 10\% FBS $(v / v), 1 \% \mathrm{P} / \mathrm{S}(v / v)$, and $2 \mu \mathrm{g} / \mathrm{mL}$ puromycin. All cells were maintained in a $5 \% \mathrm{CO}_{2}$ atmosphere at $37^{\circ} \mathrm{C}$.

\section{HEK293FT-Cre Donor Cell Line}

HEK293FT-Cre stable cell lines were made via lentiviral transduction. Lentiviral envelopes were created with VSV-G and CMV helper plasmid lipofectamine-mediated transfection (L3000008, 
Lipofectamine ${ }^{\circledR} 3000$ Transfection Reagent, Invitrogen Corp., Carlsbad, CA, USA)) in HEK293FT cells. In a $0.1 \%$ gelatin-coated 6-well plate, 500,000 HEK293FT cells/well were plated out and incubated O/N. After $24 \mathrm{~h}$, the medium was changed to DMEM (41965039, Gibco, Paisley, Scotland, UK) and supplemented with $10 \%(v / v)$ FBS. For lipofectamine transfection, a DNA sample mixture containing VSV-G $(0.5 \mu \mathrm{g}), \mathrm{CMV}(1 \mu \mathrm{g})$ and Cre plasmid $(1 \mu \mathrm{g})$ was mixed in $\mathrm{NaCl}(100 \mu \mathrm{L}, 150 \mathrm{mM}, \mathrm{pH} 7.1)$ together with $5 \mu \mathrm{g}$ P3000 ${ }^{\mathrm{TM}}$ Reagent. Subsequently, Lipofectamine ${ }^{\mathrm{TM}} 3000$ Reagent $(5 \mu \mathrm{g})$ was added to the DNA mixture and incubated together for $15 \mathrm{~min}$ at room temperature (RT). The DNA-lipid complex was added to the cells and incubatedn a $5 \% \mathrm{CO}_{2}$ environment at $37^{\circ} \mathrm{C}$. After $5-6 \mathrm{~h}$, the DNA-lipid complex containing media was aspirated and replaced with (DMEM) (Gibco, 41965039, Paisley, Scotland, UK) supplemented with $10 \%(v / v)$ FBS and $1 \%(v / v)$ P/S. After $48 \mathrm{~h}$, the supernatant was collected and centrifuged at $2000 \times g$ for $5 \mathrm{~min}$. The viral containing supernatant was added to HEK293FT cells (100,000 cells/well in a 24 -well plate seeded the day before), with polybrene. After $48 \mathrm{~h}$, the viral medium was replaced with selection medium, i.e., DMEM supplemented with $10 \%(v / v) \mathrm{FBS}, 1 \%$ $(v / v) \mathrm{P} / \mathrm{S}$, and $2 \mu \mathrm{g} / \mathrm{mL}$ puromycin.

\subsection{NV Biofabrication}

Firstly, $1-5 \times 10^{7}$ cells were harvested with $0.25 \%$ trypsin-EDTA (SLB57926) and washed with Phosphate Buffered Saline (PBS) (10010023, Gibco, Paisley, Scotland, UK). To disrupt the cells, they were resuspended in 5 mL PBS (10010023, Gibco, Paisley, Scotland, UK) and sonicated. Subsequently, the sonicated sample was recovered, transferred to the LiposoFast LF-50 (Avestin, York, UK) and extruded under compressed nitrogen through Nuclepore Track-Etch Membrane Whatman polycarbonate filters in a serial manner, i.e., starting with a $1.0 \mu \mathrm{m}$ (110610, Whatman, Maidstone, UK) followed by $0.4 \mu \mathrm{m}$ (110607, Whatman, Maidstone, UK), and finally four times through a $0.1 \mu \mathrm{m}$ Whatman ${ }^{\circledR}$ Anodisc inorganic filter membrane (6809-6012,, Whatman, Maidstone, UK), ) to yield a homogenous population size. The crude NV sample was loaded on the S400 high prep column (GE Healthcare, Uppsala, Sweden) using AKTAStart (GE Healthcare) with a $280 \mathrm{~nm}$ UV flow cell at $4{ }^{\circ} \mathrm{C}$. The NVs-containing fractions were combined, filtered $(0.45 \mu \mathrm{m})$, and concentrated with an Amicon Ultra-15 Centrifugal Filter with an Ultracel-100 membrane (UFC910024, Merck, Darmstadt, Germany). See Figure 1a for a schematic illustration of the process.

\subsection{EV Purification}

EVs were isolated by culturing 70-90\% confluent HEK293FT-Cre and A431-Cre in Opti-MEM I Reduced Serum Media (31985070, Gibco, Paisley, Scotland, UK) supplemented with 1\% P/S. Also, CPC cells were cultured in M199 medium (3115022, Gibco, Paisley, Scotland, UK) as described before [28]. After $24 \mathrm{~h}$, the conditioned medium was collected and centrifuged at $2000 \times g$ for $15 \mathrm{~min}$ to remove cell debris. The supernatant was collected, filtered $(0.45 \mu \mathrm{m})$, and concentrated with an Amicon Ultra-15 Centrifugal Filter with an Ultracel-100 membrane (UFC910024, Merck, Darmstadt, Germany). Subsequently, the concentrated conditional medium was loaded on an S400 high prep column similarly as for crude NVs. After size-exclusion chromatography (SEC), the EV-containing samples were collected, filtered $(0.45 \mu \mathrm{m})$, and concentrated with an Amicon Ultra-15 Centrifugal Filter with an Ultracel-100 membrane (UFC910024, Merck, Darmstadt, Germany).

For the Cre-loxP functionality experiment, NVs and EVs were isolated from A431-Cre and HEK293FT-Cre donor cells. These vesicles were purified accordingly to generally accepted ultracentrifugation protocol due to efficiency reasons, i.e., all samples were purified at the same time. Conditioned medium containing EVs and the crude NVs samples were centrifuged at $2000 \times g$ for $15 \mathrm{~min}$ at $4{ }^{\circ} \mathrm{C}$ to remove dead and floating cells. Subsequently, the supernatant was centrifuged at $10,000 \times g$ for $30 \mathrm{~min}$ at $4{ }^{\circ} \mathrm{C}$ to remove small vesicles and small cell debris. Finally, the supernatant was centrifuged at $100,000 \times \mathrm{g}$ for $60 \mathrm{~min}$ at $4{ }^{\circ} \mathrm{C}$ to pellet the vesicles. 


\subsection{Nanoparticle Tracking Analysis}

The size and particle concentration of EVs and NVs were assessed with nanoparticle tracking analysis (NTA) (Nanosight NS500, Malvern Panalytical Ltd., Malvern, UK). EVs and NVs were dispersed in PBS and measured in triplicate with individual measurements of $30 \mathrm{~s}$ at camera level 14. The analysis was performed with NTA software 3.3 with a minimal track length of 10 , detection threshold of 5 , and screen gain of 1.

\subsection{Western Blot}

For Western Blot analysis of vesicle protein surface markers, CPC cell lysate (CL) were dispersed in cOmplete ${ }^{\mathrm{TM}}$ Lysis-M EDTA-free (4719964001, Roche Applied Science, Mannheim, Germany) according to the manufacturer's guidelines, CPC-EVs and CPC-NVs were dispersed in RIPA buffer. Protein levels were measured with microBCA (23235, ThermoFisher Scientific, Rockford, IL, USA) and normalized to $1 \mu \mathrm{g}$ per sample. Protein samples used for $\mathrm{CD} 63$ detection were boiled at $70{ }^{\circ} \mathrm{C}$ for $10 \mathrm{~min}$. For the detection of other proteins, samples were denatured with NuPAGE ${ }^{\mathrm{TM}}$ Sample Reducing Agent $(10 \times)$ (NP0004, Invitrogen Corp., Carlsbad, CA, USA) and boiled at $70{ }^{\circ} \mathrm{C}$ for $10 \mathrm{~min}$. Samples were loaded on Bolt ${ }^{\mathrm{TM}} 4-12 \%$ Bis-Tris Plus Gel (NW04125BOX, ThermoFisher Scientific, Rockford, IL, USA) at $165 \mathrm{~V}$ for $60 \mathrm{~min}$ and transferred to PVDF membranes (IPVH00010, Merck, Darmstadt, Germany). Membranes were blocked with 5\% Bovine Serum Albumin (BSA) in Tri-Buffered Saline (TBS) for $1 \mathrm{~h}$ at RT. Subsequently, membranes were incubated with primary antibodies against Alix (177840, Abcam, Cambridge, UK), CD81 (B-11; sc-166029, Santa Cruz Biotechnology, Inc., Santa Cruz, CA, USA), Flotillin-1 antibody (ab41927, Abcam, Cambridge, UK), Calnexin (GTX101676, GeneTex, Irvine, CA, USA), $\beta$-actin (A5441, Sigma-Aldrich, Saint Louis, MO, USA), or CD63 (8219, Abcam, Cambridge, UK). Subsequently, membranes were washed and incubated for $1 \mathrm{~h}$ with appropriate secondary antibodies Goat Anti-Mouse Immunoglobulins/HRP (P0447, Dako, Santa Clara, CA, USA) or Goat Anti-Rabbit Immunoglobulins/HRP (P0448, Dako, Santa Clara, CA, USA). Proteins were visualized with chemiluminescent peroxidase substrate (CPS1120, Sigma-Aldrich, Saint Louis, MO, USA).

\subsection{Transmission Electron Microscopy}

To compare the morphology of EVs and NVs, transmission electron microscopy was performed. Carbon film copper grids (75-200 mesh) were freshly coated with the carbon coater (Edward) and $10 \mu \mathrm{L}$ of CPC-EVs and CPC-NVs samples were placed on parafilm. The carbon-coated grids were placed on top of the samples and incubated for $15 \mathrm{~min}$ at RT. Unbound vesicles were removed with PBS followed by fixation of the bound vesicles with $1 \%$ glutaraldehyde in PBS for $15 \mathrm{~min}$ at RT. PBS was removed by washing the grids on $0.2 \mu \mathrm{m}$ filtered demi water. The vesicles were stained with $2 \%$ uranyl oxalate in $0.15 \mathrm{M}$ oxalate ( $\mathrm{pH}$ 7.0) for $10 \mathrm{~min}$ at RT, followed by removal of excess stain solution with filter paper (1001090, Whatman, Maidstone UK) and MilliQ. Subsequently, the grids were incubated with $1.8 \%$ methylcellulose and $0.4 \%$ uranyl acetate for $10 \mathrm{~min}$ on ice. Excess fluid was removed and the grids were dried for $1 \mathrm{~h}$ at RT. CPC-EVs and CPC-NVs were visualized with a JEOL 3 microscope.

\subsection{Uptake Experiment}

One-hundred thousand cells/well of hfCF, CPC, HMEC, and HUVEC-GFP were plated in a 24-well plate containing $0.1 \%$ gelatin-coated cover glasses and incubated O/N. HEK293T-NVs were generated, purified with SEC, and stained with $10 \mu \mathrm{M}$ PKH-26 (PKH26GL, Sigma-Aldrich, Saint Louis, MO, USA) for 10 min at RT. Excess PKH-26 was removed by purification of the sample on an XK16 column using AKTAStart at $4{ }^{\circ} \mathrm{C}$. The PKH-26 labeled HEK293T NVs containing fractions were pooled, filtered $(0.45 \mu \mathrm{m})$, and then concentrated with a $100 \mathrm{kDa}$ MWCO Amicon spin filter (Merck). Subsequently, the amount of NVs was measured with a NTA. Then, $2 \times 10^{10} \mathrm{NVs}$ were added individually to hfCF, CPC, HMECs, and HUVECS-GFP and incubated for $24 \mathrm{~h}$ in a $5 \% \mathrm{CO}_{2}$ environment at $37^{\circ} \mathrm{C}$. After $24 \mathrm{~h}$, the medium was aspirated, the cells were washed with PBS and fixated with $4 \%$ paraformaldehyde 
(PFA) for $10 \mathrm{~min}$ and washed three times with PBS for $5 \mathrm{~min}$ at RT. The nuclei were stained with Hoechst, and the cover glasses were transferred to microscope slides on Fluoromount-G (0100-01, Southern Biotech, Birmingham, AL, USA). HEK293T-NVs uptake was visualized with Olympus BX53 microscope (magnification 20X) and confocal Zeiss LSM700 microscope (magnification 63×).

\subsection{Functional Delivery of Cre by NVs and EVs}

One-hundred thousand T47D-stoplight reporter cells/well were plated in a 24-well plate with $0.1 \%$ gelatin-coated cover glasses and incubated O/N. Then, $0.25 \mu \mathrm{g}$ TAT-CRE Recombinase (SCR508, Merck) or $0.25 \mu \mathrm{g}$ A431-Cre- and HEK293FT-Cre-derived EVs and NVs were added to T47D-stoplight reporter cells. After $72 \mathrm{~h}$, the medium was aspirated, the cells were washed with PBS, fixated with $4 \%$ PFA for $10 \mathrm{~min}$ at RT, and washed three times with PBS for $5 \mathrm{~m}$. Subsequently, the fixated cells were stained with Hoechst. The functionality of A431-Cre- and HEK293FT-Cre-derived NVs was assessed by the presence of eGFP+ T47D-stoplight reporter cells after treatment, compared to TAT-CRE Recombinase and EVs derived for the same cell-lines, using a Zeiss LSM700 microscope (magnification $63 \times$, with 405, 488, and $555 \mathrm{~nm}$ lasers used).

\subsection{Activation of ERK Signaling by CPC-EVs and -NVs}

One-hundred thousand HMEC cells/well were plated in a $0.1 \%$ gelatin-coated 48 -well plate and incubated O/N. HMECs were starved in MCDB-131 medium (Gibco) for $3 \mathrm{~h}$ and stimulated with $3 \times$ $10^{10}$ CPC-EVs or CPC-NVs for $30 \mathrm{~m}$. To assess pERK1/2 and ERK1/2 protein levels after CPC-NVs and CPC-EVs stimulation, HMEC cell lysates were obtained by suspending cells in cOmplete ${ }^{\mathrm{TM}}$ Lysis-M EDTA-free (4719964001, Roche Applied Science, Mannheim, Germany). Subsequently, protein levels were determined by microBCA assay (23235, ThermoFisher Scientific, Rockford, IL, USA) and normalized before loading the samples on Bolt ${ }^{\mathrm{TM}} 4-12 \%$ Bis-Tris Plus Gel (NW04125BOX, ThermoFisher Scientific, Rockford, IL, USA). Samples were run at $165 \mathrm{~V}$ for $50 \mathrm{~min}$ and transferred to PVDF membranes (IPVH00010, Merck, Darmstady, Germany). Membranes were blocked for $1 \mathrm{~h}$ with 5\% BSA in TBS and incubated with primary antibodies phospho-p44/42 MAPK (pERK1/2) (, 9101S, Cell Signaling, Beverly, CA, USA) and p44/42 MAPK (Erk1/2) (9102S, Cell Signaling, Beverly, CA, USA). Subsequently, membranes were washed with TBS and incubated with Goat Anti-Rabbit Immunoglobulins/HRP (P0448, Dako, Santa Clara, CA, USA) secondary antibody. Proteins were visualized with chemiluminescent peroxidase substrate (CPS1120, Sigma-Aldrich, Saint Louis, MO, USA).

\subsection{Comparative Analysis NV and EV Production}

Vesicle number and protein concentration of NVs and EVs recovered from $1 \times 10^{7} \mathrm{CPC}$ were determined with NTA and microBCA (23235, ThermoFisher Scientific, Rockford, IL, USA), respectively. The total number of particles and protein content was calculated by multiplying the final volumes of $\mathrm{NVs}$ or EVs by the measured particle and protein concentrations.

\subsection{Statistical Analysis}

All statistical analysis was performed with GraphPad Prism 7 using one-way ANOVA with Bonferroni correction for multiple comparisons. Data is presented as mean \pm SEM. A $p$-value $<0.05$ was considered as significant difference.

\section{Results}

\subsection{Production}

\subsubsection{Process Characterization}

To produce NVs, cells were sonicated, sequentially extruded through membranes with decreasing pore size (subsequently 1, 0.4, and $0.1 \mu \mathrm{m}$ ) using compressed nitrogen (10-40 bar), which was 
followed by size-exclusion chromatography (SEC) purification (Figure 1a) [28]. NTA showed that the nonhomogenous populations yielded by sonication are formed into a homogenous NV peak-diameters between $\sim 50-200 \mathrm{~nm}$ through sequential extrusion through microfilters (Figure 1b).

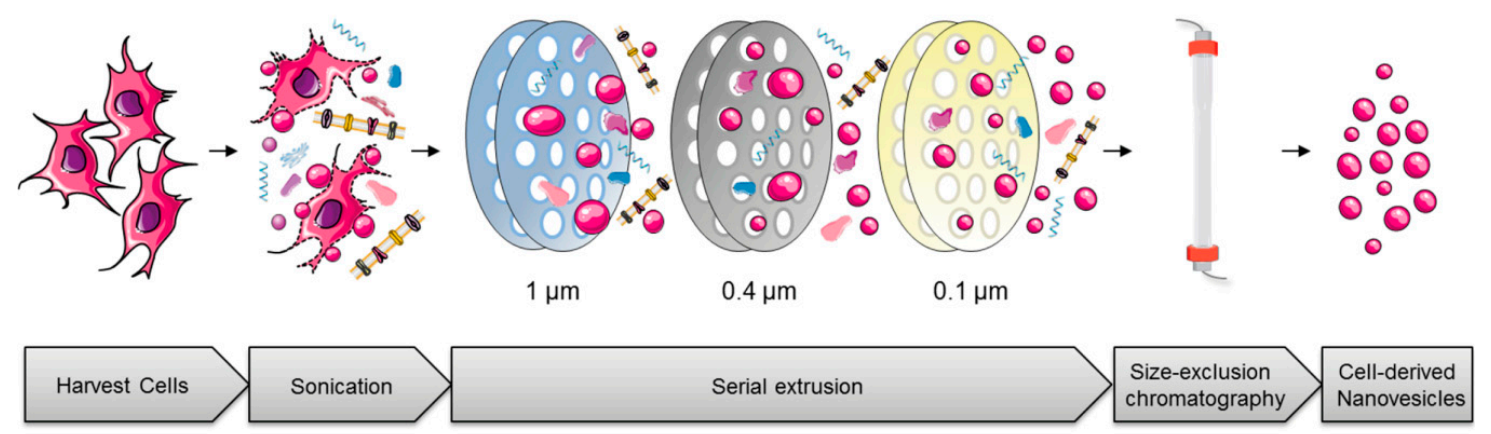

(a)
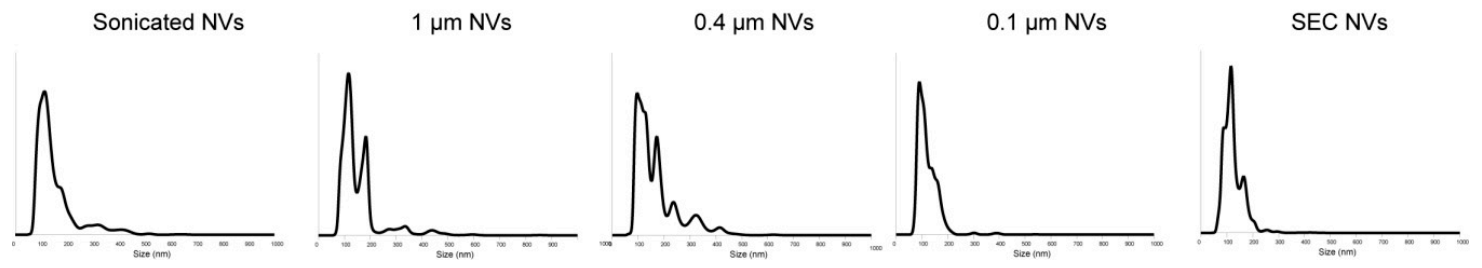

(b)

Figure 1. Establishment of the biofabrication of nanovesicles (NVs) in cardiac progenitor cells (CPCs). (a) Schematic illustration of NV generation and purification. (b) NTA showed that the extrusion process through filters yielded a homogenous NVs population with a $\sim 50-200 \mathrm{~nm}$ peak diameter. The size of the NVs became more homogenous after each step. These figures are representative results of at least four independent experiments.

\subsubsection{Characterization of CPC-NVs and CPC-EVs}

CPC-NVs and CPC-EVs were characterized for size, morphology, and protein surface markers. NTA analysis of purified CPC-EVs and CPC-NVs showed a comparable size distribution between $\sim 50-200 \mathrm{~nm}$, with a mean diameter $\pm 126 \mathrm{~nm}$ (Figure 2a,b). Transmission electron microscopy showed a 'cup-shaped' morphology of CPC-NVs, which was comparable to the morphology of CPC-EVs (Figure 2c). Western blot analysis demonstrated that CPC-NVs are enriched for integral caveolae-associated membrane protein flotillin-1, an integral protein of the endoplasmic reticulum calnexin and $\beta$-actin. In contrast, CPC-EVs are enriched for tetraspanins CD81 and CD63 and the multivesicular body protein Alix (Figure 2d). Additionally, NTA of two individual CPC-NV preparations stored at $-80{ }^{\circ} \mathrm{C}$ showed that $\mathrm{CPC}-\mathrm{NV}$ s slightly increase in size and decrease in concentration over time (Figure S4). 


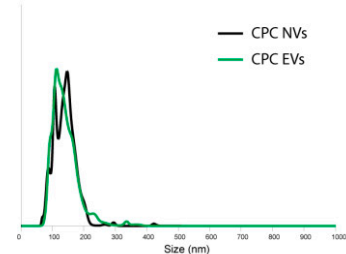

(a)

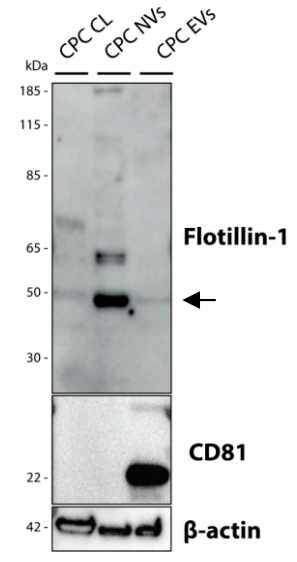

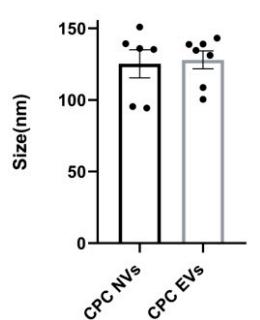

(b)
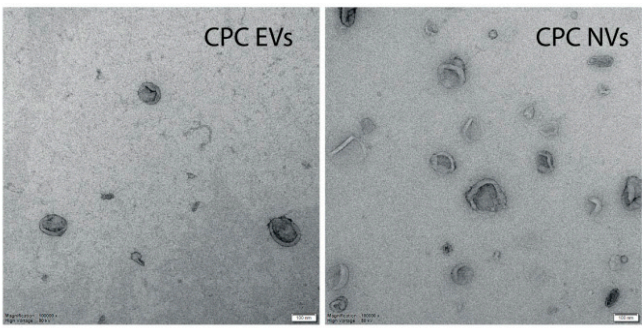

(c)

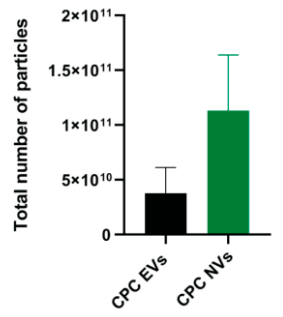

(e)

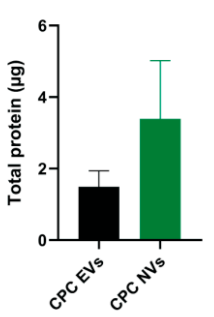

(f)

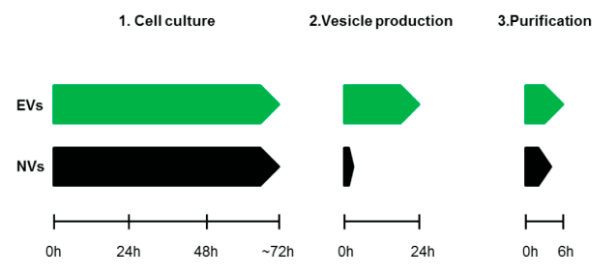

(g)

Figure 2. Comparative analysis of CPC-EVs and CPC-NVs characteristics and production process. NTA analysis showed that CPC-EVs and CPC-NVs have a comparable peak diameter between (a) $\sim 50-200 \mathrm{~nm}$, and (b) a mean diameter $\pm 126 \mathrm{~nm}$. (c) Transmission electron microscopy showed a similar 'cup-shaped' morphology for both CPC-EVs and NVs. Scale bar $=100 \mathrm{~nm}$ (d) Western blot analysis of 1 $\mu \mathrm{g}$ CPC cell lysate (CL), CPC-NVs and CPC-EVs show that CPC-NVs are enriched with flotillin-1 and calnexin. In contrast, CPC-EVs are enriched with EV protein markers CD81, CD63, and Alix. (e) Total number of particles and (f) total protein content of CPC-EVs and CPC-NVs derived from $1 \times 10^{7} \mathrm{CPC}$ show that NVs are produced in a higher quantity than EVs when derived from the same cell density $(n=2)$. (g) Schematic overview of the production and purification timeline of EVs and NVs shows that $\mathrm{NV}$ production is more efficient compared to EV production. Data expressed as mean $\pm \mathrm{SEM}$. EVs $=$ extracellular vesicles.

To assess if NV biofabrication is more efficient than EV production, we determined the time needed to complete either production process and measured the quantity of NVs and EVs that were recovered from equal cell numbers. When vesicles were derived from $1 \times 10^{7} \mathrm{CPC}$, the total yield in terms of the total number of particles and protein was higher for NV biofabrication compared to EV production (Figure 2e,f). To yield an approximately a similar amount of a total number of particles, CPC-EVs needs to be derived from 11-fold higher cell density compared to CPC-NVs (Figure S1). Also, the initial cell density prior to NV biofabrication determines the total number of particles recovered after SEC (Figure S2). Furthermore, NV biofabrication is more time-efficient and less laborious with respect to the production and purification phase (Figure 2g). Compared to EVs production, $\mathrm{NV}$ biofabrication requires less time, materials, and labor intensity for the upscaling of cell culture and eliminates a concentrating step to prepare conditioned medium for SEC. These observations show that after SEC, CPC-NVs are produced more efficiently (Figure 2e-g) and share a similar size-distribution and morphology as CPC-EVs (Figure 2b,c). However, CPC-NVs do not contain standard EV protein markers, indicating that they represent a different vesicle type. 


\subsection{Functionality}

\subsubsection{NVs Uptake}

To confirm that NVs could be internalized by cells, we labeled HEK293FT-NVs with a red fluorescent PKH-26 dye and incubated them for $24 \mathrm{~h}$ with different target cells, including two types of endothelial cells (HMECs and HUVECs-GFP), fibroblasts (hfCF), and cardiac progenitor cells (CPCs). The internalization of fluorescent-labeled NVs by endothelial and cardiac cells was confirmed by microscopy analysis (Figure $3 a, b$ ) and resulted in a peri-nuclear pattern, as we have seen before for EVs [13].
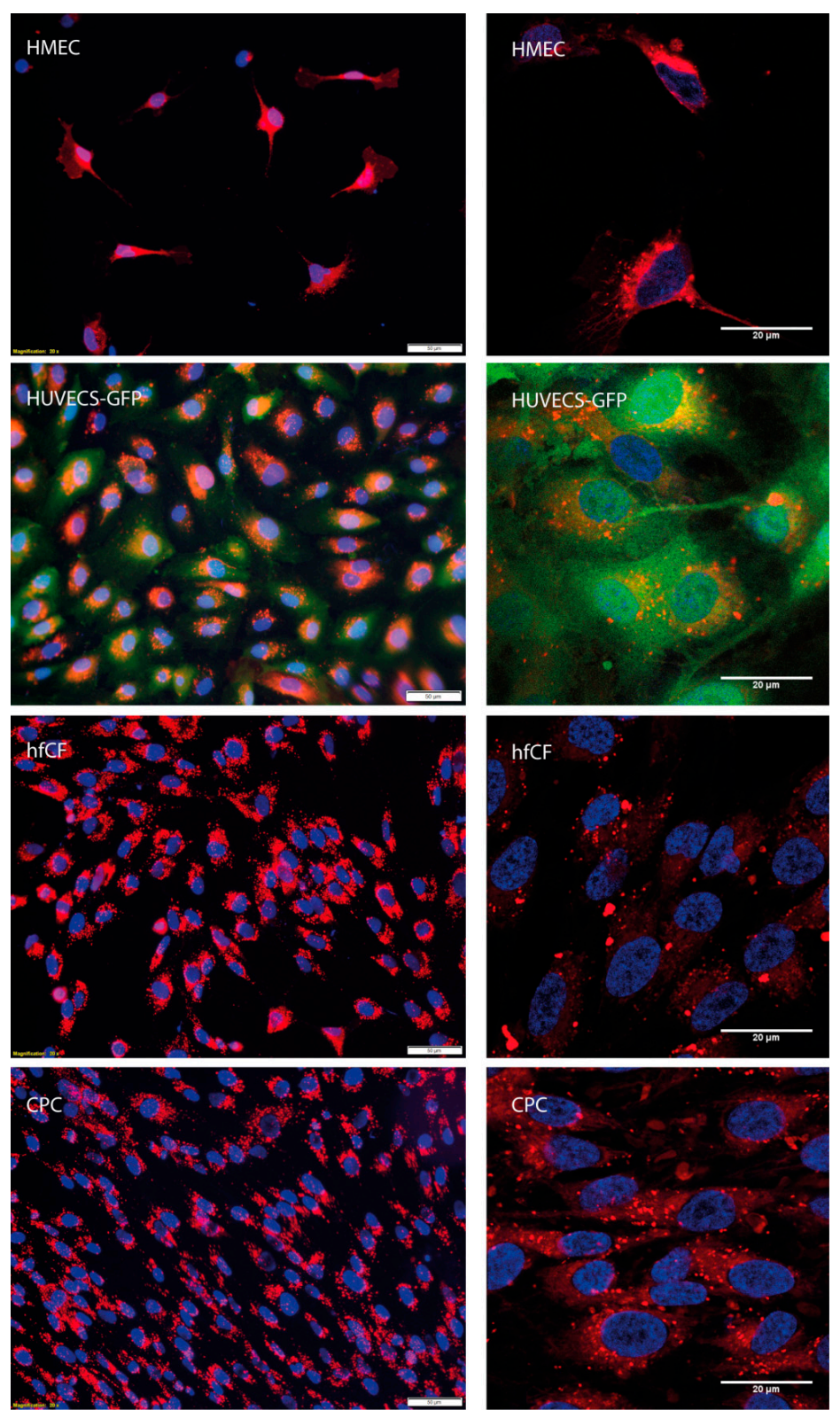

(a)

(b)

Figure 3. CPC-NV uptake by HMECs, HUVECS-GFP, hfCF, and CPCs. (a) Microscopic analysis by Olympus BX53 microscope. Scale bar $=50 \mu \mathrm{m}$. (b) Confocal microscopy by LSM Zeiss 700. Scale bar $=20 \mu \mathrm{m}$. (a) and (b) demonstrated internalization of PKH-26 positive NVs upon incubation of $2 \times$ $10^{10} \mathrm{NVs}$ with HMECs, HUVECS-GFP, hfCF, and CPC for $24 \mathrm{~h}$. NVs = PKH-26 (red) and nucleus = Hoechst (blue). 


\subsubsection{NVs Functionally Deliver Biomolecules to Target Cells}

To assess if NVs can deliver functional biomolecules to recipient cells, we used the Cre-loxP method to investigate the vesicle-mediated transfer of cargo to a T47D-stoplight reporter cell line. Upon delivery of Cre biomolecules (mRNA or protein) to the reporter cell line, DsRed + reporter cells will be recombined to eGFP+ cells when the stop-codon is removed between loxP sites (Figure 4a). Confocal microscopy images confirmed the presence of eGFP+ reporter cells when TAT-Cre recombinase, $\mathrm{Cre}+-\mathrm{EVs}$, or Cre+-NVs were introduced (Figure $4 \mathrm{~b}, \mathrm{c}$ ). Although the absolute number of eGFP+ reporter cells was low, we could reproducibly detect them for all these conditions. No eGFP+ reporter cells were observed when T47D-stoplight reporter cells were treated with vesicles derived from a non-Cre donor cell HEK293FT (data not shown). NTA characterization of Cre+-NVs and EVs showed that these vesicles share similar sizes (Figure S3a,b). These observations indicate that NVs and EVs have a similar size and are both capable of transferring functional biomolecules from donor cells to recipient cells.

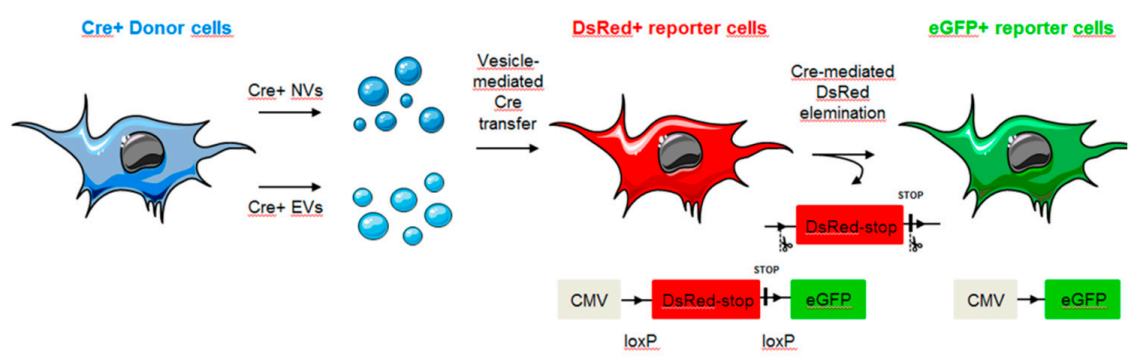

(a)

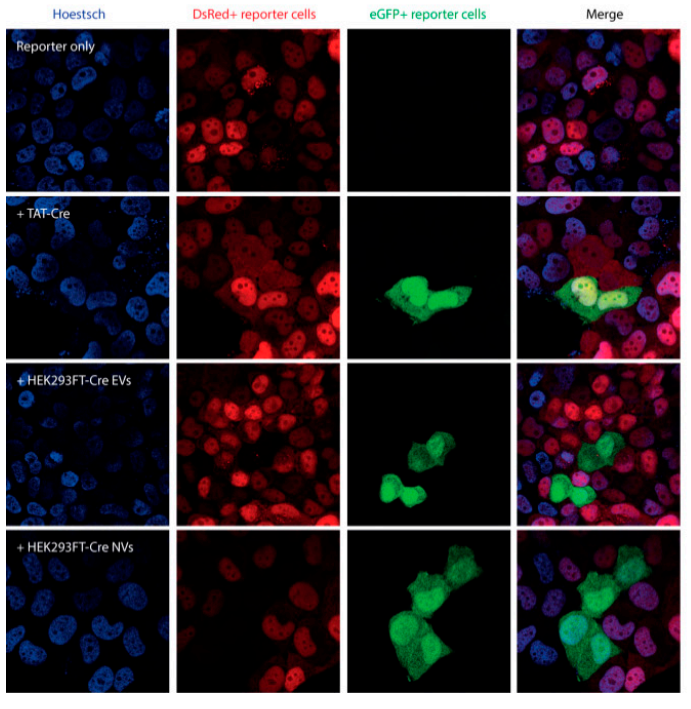

(b)

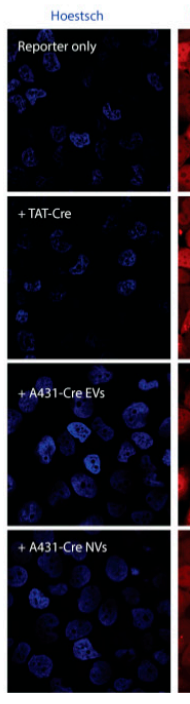

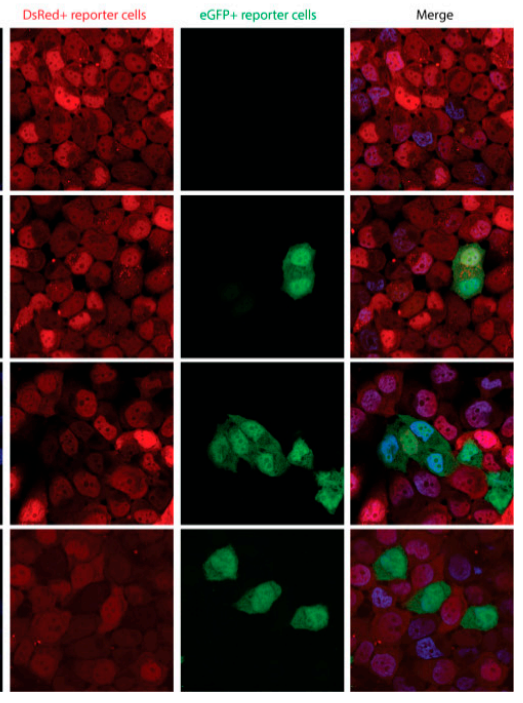

(c)

Figure 4. Cre+-EVs and Cre+-NVs transfer of Cre-recombinase mRNA or protein to T47D Cre-reporter cells. (a) Schematic illustration of Cre+ vesicle-transfer to reporter cells: DsRed + reporter cells will be recombined to eGFP+ cells when the stop-codon is removed between indicated loxP sites. Visualization of functional effect induced by TAT-Cre recombinase, Cre+-EVs andCre+-NVs derived from HEK293FT-Cre (b), and A431-Cre donor cells (c).

\subsubsection{CPC-NVs and EVs Induce Phosphorylation of ERK1/2}

We have previously used CPC-EVs-induced ERK1/2 phosphorylation in HMEC-1 as a functional outcome measurement [28]. Similar to CPC-EVs, CPC-NVs induced phosphorylation of ERK1/2, as determined by western blot analyses (Figure 5a) and quantified using ImageJ (Figure 4a). 
These observations show that CPC-NVs, similar to EVs, can activate intracellular pro-survival signaling pathways.

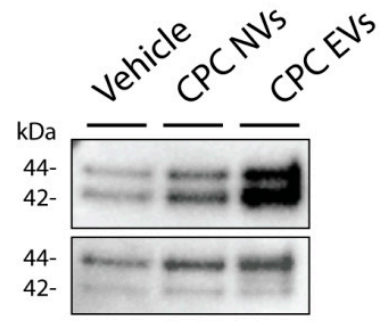

(a)

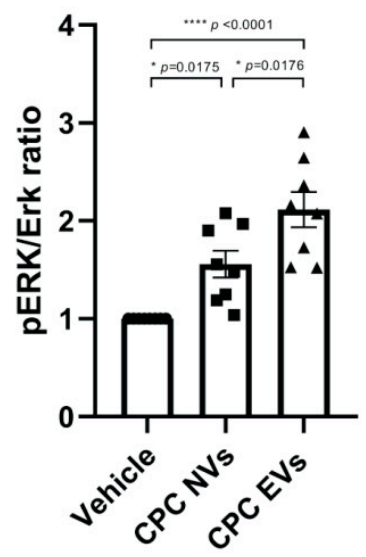

(b)

Figure 5. CPC-NVs and CPC-EVs induce phosphorylation of ERK1/2: (a) Protein levels of pERK 1/2 and ERK1/2 determined by western blot. (b) Quantification of ratio phosphorylated ERK1/2 to ERK1/2 level. Mean results of at least six independent experiments \pm SEM.

\section{Discussion}

EVs have gained increasing attention over the years for their application as either a drug delivery system or as cell-free therapeutic. However, despite this potential, EVs' clinical translation is limited by a lack of efficient, scalable, and cost-effective production methods. In this study, we aimed to investigate the biofabrication of NVs as an alternative production method to make EV mimetics.

NV biofabrication is based on the spontaneous self-assembly of phospholipids, which are abundantly present in the cell membrane $[29,30]$. Phospholipids are amphiphiles, i.e., they have a hydrophobic tail and hydrophilic headgroup, which enables the cell membrane components to self-assemble into vesicles in an aqueous solution via minimalization of line energy along the free edge of membrane patches. As a result, planar bilayer membrane curves into a spherical cap, ultimately resulting in a closed nanovesicle [29,30]. We hypothesized that NV biofabrication, during which the released cell membranes will self-assemble into cell-derived nanovesicles while encapsulating the released cytosolic material during the self-assembly process, is a more efficient process than EV production to create EV mimetics.

In this study, we demonstrated that NV biofabrication is a scalable, time-efficient, and cost-effective method capable of yielding cell-derived nanosized vesicles in higher quantities when compared to EV isolation from the same cell density (Figure 2e,f). Furthermore, NVs are produced and purified in a more time-efficient manner compared to EVs (Figure $2 \mathrm{~g}$ ). For both vesicle productions, the most time-consuming step is for cells to reach the desired cell density. However, the upscaling of cells for EV production is more labor-intensive and cost-ineffective compared to production for NVs by biofabrication. After the cell growth phase, vesicle production is the next rate-determining step. When cells are cultured without FBS, EVs are typically isolated after 24-72 h.

In contrast, NVs can be produced within $2 \mathrm{~h}$ and when needed once the desired cell density is reached. This convenience makes NVs fabrication a faster alternative to obtain nanosized vesicles with EV-like size and morphology. Additionally, NV biofabrication is a more cost-effective method, since the production phase requires neither additional culture medium nor labor-intensive efforts to isolate and prepare EVs prior to SEC purification. These observations together demonstrate that the generation of NVs is a faster and therefore more efficient method for preparing cell-derived nanovesicles, as compared to the production of natural EVs. 
When CPC-NVs were stored at $-80{ }^{\circ} \mathrm{C}$, we observe a small increase in size and decrease in concentration. These observations may be explained by $\mathrm{NVs}^{\prime}$ membrane fusion or adherence to the Eppendorf tubes. Hence, NV storage and stability need to be further characterized. Additionally, the current understanding of EVs storage and stability remains unclear. Hence, EVs and NVs storage and preservation need to extensively investigated in future studies for their therapeutic application [31].

NVs have a comparable size distribution $(\sim 50-200 \mathrm{~nm})$ and morphology as EVs. Interestingly, while CPC-NVs are enriched for flotillin-1 and calnexin, CPC-EVs are enriched in EV protein markers CD63, CD81, and Alix. NV enrichment of flotillin-1 and calnexin and not with endosomal proteins suggests that CPC-NVs are derived from plasma- and endoplasmic reticulum membranes while CPC-EVs may to a large extent be derived from endosomes. Thus, CPC-EVs and CPC-NVs are two different types of vesicles. These observations are in contradiction with previous studies which show that U937- and adipose stem cell-derived nanovesicles, produced via spin-cups with SEC purification or microfilters followed by centrifugation and Opti-Prep gradient purification, respectively, are enriched with tetraspanin- and multivesicular body proteins [21,32].

Furthermore, the study of Park et al. (2019) demonstrated that mesenchymal stromal cell-derived nanovesicles are enriched with CD81, flotillin-1, and $\beta$-actin, while we only found CPC-EVs to be enriched with CD81 [33]. Taken together, these contradictions may indicate that the production of alternative cell-derived nanovesicles is dependent on cell type, production or purification methods. However, to assess the exact difference between NVs and EVs, in-depth proteomics and RNAomics would provide more insight into the differences or similarities between these vesicles. Nevertheless, NVs can be internalized by various cell lines in vitro, suggesting that the lack of the investigated protein markers does not affect the ability of cells to internalize such vesicles.

EVs deliver cytosolic content of their donor cells to recipient cells that lead to changes in the behavior of cells $[34,35]$. We showed that NVs are also capable of delivering functional biological material to recipient cells, which induces a cellular activation. Yet, the efficiency of functional transfer via NVs and EVs remains to be determined. Nonetheless, eGFP+ reporter cells are observed when treated with Cre+-EVs and Cre+-NVs derived from different Cre+ donor cells. Importantly, we used donor cells stably expressing Cre, allowing us to study only cell-derived vesicle-mediated transfer of Cre cytosolic RNA (functional RNA) and protein, without the interference of lipid-based carriers that may end up in NVs/EVs and affect their behavior. [36-39].

The observation of eGFP+ reporter cells demonstrates $\mathrm{NVs}^{\prime}$ potential to be developed as a potential drug delivery system for biotherapeutics produced in cells. Additionally, the cell membrane of the cells used for NV biofabrication can be modified using molecular engineering techniques to express surface molecules. Consequently, these engineered nanovesicles can mediate a specific biological function or neutralize active biological molecules in circulation. Also, the cell membrane can be engineered to contain ligands that recognize specific targets or tissue [40]. Thus, generating NVs from these cell lines could enable NVs to facilitated targeted delivery [1]. Taken together, biofabrication of NVs combined with molecular engineering cells to contain surface proteins or targeting ligand opens up a wide variety of possibilities to use NVs for (targeted) delivery platforms.

Finally, functional assessment of CPC-NVs and CPC-EVs demonstrated that both types of vesicles are capable of activating pro-survival pathways. However, more in vitro functional assays, e.g., apoptosis or proliferation assays, as well as in vivo studies in myocardial infarct mouse models, are required to establish the therapeutic value of CPC-NVs as an alternative for CPC-EVs in regenerative medicine. Furthermore, there remains a lack of understanding regarding the stability and safety of EVs and NVs. Consequently, subsequent studies should be done to address CPC-EVs and CPC-NVs' stability, toxicity, and potential immunogenicity [18].

\section{Conclusions}

In the present work, we demonstrated that biofabrication of NVs is a scalable, time-efficient, and cost-effective production method to yield more nanoparticles from the same cell density when 
compared to EVs. Furthermore, we show that CPC-NVs and CPC-EVs share a comparable size and morphology. However, we also show that they are two different types of vesicles regarding their composition of their membrane markers. Yet, NVs can be taken up by hfCF, CPC, and endothelial cells. Finally, we observed the functionality of NVs in delivering Cre-recombinase mRNA or protein as well as activating ERK1/2 signaling. Taken together, we present here an effective method to produce cell-derived nanovesicles with similar functional properties as EVs. Therefore, NVs hold the potential to be employed as a therapeutic agent in regenerative medicine. Additionally, biofabrication of NVs gives rise to new opportunities to create targeted drug delivery systems by functionalizing cell membranes or therapeutic loading cargo inside NVs.

Supplementary Materials: The following are available online at http://www.mdpi.com/2073-4409/8/12/1509/s1, Figure S1: An 11-fold higher cell density is needed for EVs production compared to NV biofabrication to yield the same amount of particles, Figure S2: Total number of NVs recovered after SEC is dependent on initial cell density, Figure S3: Characterization of Cre+-EVs and Cre+-NVs, Figure S4: Characterization of CPC-NVs stored at $-80^{\circ} \mathrm{C}$ over time from two individual isolations.

Author Contributions: Conceptualization, Z.L. and J.P.G.S.; methodology, N.F.I., E.A.M. and Z.L.; formal analysis, N.F.I.; investigation, N.F.I., Z.L., E.A.M., A.K.D., L.J., and R.M.S.; data curation, N.F.I., E.A.M. and Z.L.; writing-original draft preparation, N.F.I.; writing-review and editing, N.F.I., Z.L., P.V., E.A.M., L.J., A.K.D., and J.P.G.S.; visualization, N.F.I.; supervision, Z.L. and J.P.G.S.; project administration, Z.L.; funding acquisition, J.P.G.S., Z.L., P.V., and R.M.S.

Funding: This work was supported by the Project EVICARE (No. 725229) of the European Research Council (ERC) to J.P.G.S., co-funded by the Project SMARTCARE-II of the BioMedicalMaterials institute to J.P.G.S., the ZonMw-TAS program (No. 116002016) to J.P.G.S./Z.L., PPS grant (No. 2018B014) to J.P.G.S./P.V/Z.L, the Dutch Ministry of Economic Affairs, Agriculture and Innovation and the Netherlands CardioVascular Research Initiative (CVON): the Dutch Heart Foundation to J.P.G.S., Dutch Federations of University Medical Centers, the Netherlands Organization for Health Research and Development, and the Royal Netherlands Academy of Sciences.

Acknowledgments: The authors would like to thank Cor van Seinen, Iris van Adrichem, Jan Strecker, Peter Bokern, Marieke Roefs, and Simon van de Wakker for their experimental support. Thanks to Corlinda ten Brink and the Cell Microscopy Core of the Department of Cell Biology at the University Medical Center Utrecht in the Netherlands for their support and use of imaging equipment. Marie-Jose Goumans, LUMC, is acknowledged for providing the CPC cell populations. Schematic illustrations of the NV biofabrication process and studying Cre-mediated transfer were created and adapted from Servier Medical Art (www.servier.co.uk/content/serviermedical-art). Servier Medical Art is licensed under a Creative Commons Attribution 3.0 Unported License (http://creative-commons.org/licenses/by/3.0).

Conflicts of Interest: P.V. serves on the scientific advisory board of Evox Therapeutics.

\section{References}

1. Wiklander, O.P.B.; Brennan, M.Á.; Lötvall, J.; Breakefield, X.O.; Andaloussi, S.E.L. Advances in therapeutic applications of extracellular vesicles. Sci. Transl. Med. 2019, 11, eaav8521. [CrossRef] [PubMed]

2. Vader, P.; Mol, E.A.; Pasterkamp, G.; Schiffelers, R.M. Extracellular vesicles for drug delivery. Adv. Drug Deliv. Rev. 2016, 106, 148-156. [CrossRef] [PubMed]

3. Time to deliver. Nat. Biotechnol. 2014, 32, 961. [CrossRef] [PubMed]

4. Zhang, L.; Gu, F.X.; Chan, J.M.; Wang, A.Z.; Langer, R.S.; Farokhzad, O.C. Nanoparticles in medicine: Therapeutic applications and developments. Clin. Pharmacol. Ther. 2008, 83, 761-769. [CrossRef] [PubMed]

5. Taheri, B.; Soleimani, M.; Fekri Aval, S.; Esmaeili, E.; Bazi, Z.; Zarghami, N. Induced pluripotent stem cell-derived extracellular vesicles: A novel approach for cell-free regenerative medicine. J. Cell. Physiol. 2019, 234, 8455-8464. [CrossRef] [PubMed]

6. Vishnubhatla, I.; Corteling, R.; Stevanato, L.; Hicks, C.; Sinden, J. The development of stem cell-derived exosomes as a cell-free regenerative medicine. J. Circ. Biomarkers 2014, 3, 2-3. [CrossRef]

7. Camussi, G.; Deregibus, M.C.; Cantaluppi, V. Role of stem-cell-derived microvesicles in the paracrine action of stem cells. Biochem. Soc. Trans. 2013, 41, 283-287. [CrossRef]

8. Arslan, F.; Lai, R.C.; Smeets, M.B.; Akeroyd, L.; Choo, A.; Aguor, E.N.E.; Timmers, L.; van Rijen, H.V.; Doevendans, P.A.; Pasterkamp, G.; et al. Mesenchymal stem cell-derived exosomes increase ATP levels, decrease oxidative stress and activate PI3K/Akt pathway to enhance myocardial viability and prevent adverse remodeling after myocardial ischemia/reperfusion injury. Stem Cell Res. 2013, 10, 301-312. [CrossRef] 
9. Timmers, L.; Lim, S.K.; Hoefer, I.E.; Arslan, F.; Lai, R.C.; van Oorschot, A.A.M.; Goumans, M.J.; Strijder, C.; Sze, S.K.; Choo, A. Human mesenchymal stem cell-conditioned medium improves cardiac function following myocardial infarction. Stem Cell Res. 2011, 6, 206-214. [CrossRef]

10. Lai, R.C.; Chen, T.S.; Lim, S.K. Mesenchymal stem cell exosome: A novel stem cell-based therapy for cardiovascular disease. Regen. Med. 2011, 6, 481-492. [CrossRef]

11. Timmers, L.; Lim, S.K.; Arslan, F.; Armstrong, J.S.; Hoefer, I.E.; Doevendans, P.A.; Piek, J.J.; El Oakley, R.M.; Choo, A.; Lee, C.N. Reduction of myocardial infarct size by human mesenchymal stem cell conditioned medium. Stem Cell Res. 2008, 1, 129-137. [CrossRef] [PubMed]

12. Barile, L.; Lionetti, V.; Cervio, E.; Matteucci, M.; Gherghiceanu, M.; Popescu, L.M.; Torre, T.; Siclari, F.; Moccetti, T.; Vassalli, G. Extracellular vesicles from human cardiac progenitor cells inhibit cardiomyocyte apoptosis and improve cardiac function after myocardial infarction. Cardiovasc. Res. 2014, 103, 530-541. [CrossRef] [PubMed]

13. Vrijsen, K.R.; Sluijter, J.P.G.; Schuchardt, M.W.L.; Van Balkom, B.W.M.; Noort, W.A.; Chamuleau, S.A.J.; Doevendans, P. Cardiomyocyte progenitor cell-derived exosomes stimulate migration of endothelial cells. J. Cell. Mol. Med. 2010, 14, 1064-1070. [CrossRef] [PubMed]

14. Lamichhane, T.N.; Sokic, S.; Schardt, J.S.; Raiker, R.S.; Lin, J.W.; Jay, S.M. Emerging roles for extracellular vesicles in tissue engineering and regenerative medicine. Tissue Eng. Part. B Rev. 2014, 21, 45-54. [CrossRef] [PubMed]

15. Mol, E.A.; Goumans, M.-J.; Sluijter, J.P.G. Cardiac progenitor-cell derived exosomes as cell-free therapeutic for cardiac repair. Adv. Exp. Med. Biol. 2017, 998, 207-219.

16. Segers, V.F.M.; Lee, R.T. Stem-cell therapy for cardiac disease. Nature 2008, 451, 937-942. [CrossRef]

17. Steinhauser, M.L.; Lee, R.T. Regeneration of the heart. EMBO Mol. Med. 2011, 3, 701-712. [CrossRef]

18. Sluijter, J.P.G.; Davidson, S.M.; Boulanger, C.M.; Buzas, E.I.; De Kleijn, D.P.V.; Engel, F.B.; Giricz, Z.; Hausenloy, D.J.; Kishore, R.; Lecour, S. Extracellular vesicles in diagnostics and therapy of the ischaemic heart: Position Paper from the Working Group on Cellular Biology of the Heart of the European Society of Cardiology. Cardiovasc. Res. 2017, 114, 19-34. [CrossRef]

19. Lu, M.; Xing, H.; Yang, Z.; Sun, Y.; Yang, T.; Zhao, X.; Cai, C.; Wang, D.; Ding, P. Recent advances on extracellular vesicles in therapeutic delivery: Challenges, solutions, and opportunities. Eur. J. Pharm. Biopharm. 2017, 119, 381-395. [CrossRef]

20. Jo, W.; Kim, J.; Yoon, J.; Jeong, D.; Cho, S.; Jeong, H.; Yoon, Y.J.; Kim, S.C.; Gho, Y.S.; Park, J. Large-scale generation of cell-derived nanovesicles. Nanoscale 2014, 6, 12056-12064. [CrossRef]

21. Goh, W.J.; Zou, S.; Ong, W.Y.; Torta, F.; Alexandra, A.F.; Schiffelers, R.M.; Storm, G.; Wang, J.-W.; Czarny, B.; Pastorin, G. Bioinspired Cell-Derived Nanovesicles versus Exosomes as Drug Delivery Systems: A Cost-Effective Alternative. Sci. Rep. 2017, 7, 14322. [CrossRef] [PubMed]

22. Ban, J.-J.; Lee, M.; Im, W.; Kim, M. Low pH increases the yield of exosome isolation. Biochem. Biophys. Res. Commun. 2015, 461, 76-79. [CrossRef] [PubMed]

23. Garcia, N.A.; Ontoria-Oviedo, I.; González-King, H.; Diez-Juan, A.; Sepúlveda, P. Glucose Starvation in Cardiomyocytes Enhances Exosome Secretion and Promotes Angiogenesis in Endothelial Cells. PLoS ONE 2015, 10, e0138849. [CrossRef] [PubMed]

24. Savina, A.; Furlán, M.; Vidal, M.; Colombo, M.I. Exosome release is regulated by a calcium-dependent mechanism in K562 cells. J. Biol. Chem. 2003, 278, 20083-20090. [CrossRef]

25. Kapustin, A.N.; Chatrou, M.L.; Drozdov, I.; Zheng, Y.; Davidson, S.M.; Soong, D.Y.H.; Furmanik, M.; Sanchis, P.; de Rosales, R.T.; Alvarez-Hernandez, D. Vascular smooth muscle cell calcification is mediated by regulated exosome secretion. Circ. Res. 2015, 116, 1312-1323. [CrossRef]

26. Smits, A.M.; van Vliet, P.; Metz, C.H.; Korfage, T.; Sluijter, J.P.G.; Doevendans, P.A.; Goumans, M.-J. Human cardiomyocyte progenitor cells differentiate into functional mature cardiomyocytes: An in vitro model for studying human cardiac physiology and pathophysiology. Nat. Protoc. 2009, 4, 232-243. [CrossRef]

27. Van Mil, A.; Grundmann, S.; Goumans, M.-J.; Lei, Z.; Oerlemans, M.I.; Jaksani, S.; Doevendans, P.A.; Sluijter, J.P.G. MicroRNA-214 inhibits angiogenesis by targeting Quaking and reducing angiogenic growth factor release. Cardiovasc. Res. 2012, 93, 655-665. [CrossRef]

28. Mol, E.A.; Goumans, M.J.; Doevendans, P.A.; Sluijter, J.P.G.; Vader, P. Higher functionality of extracellular vesicles isolated using size-exclusion chromatography compared to ultracentrifugation. Nanomedicine 2017, 13, 2061-2065. [CrossRef] 
29. Huang, C.; Quinn, D.; Sadovsky, Y.; Suresh, S.; Hsia, K.J. Formation and size distribution of self-assembled vesicles. Proc. Natl. Acad. Sci. USA 2017, 114, 2910-2915. [CrossRef]

30. Yoon, J.; Jo, W.; Jeong, D.; Kim, J.; Jeong, H.; Park, J. Generation of nanovesicles with sliced cellular membrane fragments for exogenous material delivery. Biomaterials 2015, 59, 12-20. [CrossRef]

31. Jeyaram, A.; Jay, S.M. Preservation and storage stability of extracellular vesicles for therapeutic applications. AAPS J. 2018, 20, 1. [CrossRef] [PubMed]

32. Kim, Y.-S.; Kim, J.-Y.; Cho, R.; Shin, D.-M.; Lee, S.W.; Oh, Y.-M. Adipose stem cell-derived nanovesicles inhibit emphysema primarily via an FGF2-dependent pathway. Exp. Mol. Med. 2017, 49, e284. [CrossRef] [PubMed]

33. Park, K.-S.; Svennerholm, K.; Shelke, G.V.; Bandeira, E.; Lässer, C.; Jang, S.C.; Chandode, R.; Gribonika, I.; Lötvall, J. Mesenchymal stromal cell-derived nanovesicles ameliorate bacterial outer membrane vesicle-induced sepsis via IL-10. Stem Cell Res. Ther. 2019, 10, 231. [CrossRef]

34. Zomer, A.; Steenbeek, S.C.; Maynard, C.; van Rheenen, J. Studying extracellular vesicle transfer by a Cre-loxP method. Nat. Protoc. 2016, 11, 87-101. [CrossRef] [PubMed]

35. Zomer, A.; Maynard, C.; Verweij, F.J.; Kamermans, A.; Schäfer, R.; Beerling, E.; Schiffelers, R.M.; de Wit, E.; Berenguer, J.; Ellenbroek, S.I.J. In vivo imaging reveals extracellular vesicle-mediated phenocopying of metastatic behavior. Cell 2015, 161, 1046-1057. [CrossRef]

36. Maugeri, M.; Nawaz, M.; Papadimitriou, A.; Angerfors, A.; Camponeschi, A.; Na, M.; Hölttä, M.; Skantze, P.; Johansson, S.; Sundqvist, M. Linkage between endosomal escape of LNP-mRNA and loading into EVs for transport to other cells. Nat. Commun. 2019, 10,1-15. [CrossRef]

37. Ridder, K.; Keller, S.; Dams, M.; Rupp, A.-K.; Schlaudraff, J.; Del Turco, D.; Starmann, J.; Macas, J.; Karpova, D.; Devraj, K. Extracellular vesicle-mediated transfer of genetic information between the hematopoietic system and the brain in response to inflammation. PLoS Biol. 2014, 12, e1001874. [CrossRef]

38. Usman, W.M.; Pham, T.C.; Kwok, Y.Y.; Vu, L.T.; Ma, V.; Peng, B.; San Chan, Y.; Wei, L.; Chin, S.M.; Azad, A. Efficient RNA drug delivery using red blood cell extracellular vesicles. Nat. Commun. 2018, 9, 2359. [CrossRef]

39. Lin, Y.; Wu, J.; Gu, W.; Huang, Y.; Tong, Z.; Huang, L.; Tan, J. Exosome-liposome hybrid nanoparticles deliver CRISPR/Cas9 system in MSCs. Adv. Sci. 2018, 5, 1700611. [CrossRef]

40. De Jong, O.G.; Kooijmans, S.A.A.; Murphy, D.E.; Jiang, L.; Evers, M.J.W.; Sluijter, J.P.G.; Vader, P.; Schiffelers, R.M. Drug Delivery with Extracellular Vesicles: From Imagination to Innovation. Acc. Chem. Res. 2019, 52, 1761-1770. [CrossRef] 